\title{
Crisis e intercooperación. La experiencia uruguaya
}

\author{
Siegbert Rippe \\ Doctor en Derecho y Ciencias Sociales, Profesor Emérito \\ y Catedrático de Derecho Comercial \\ (Universidad de la República - Uruguay)
}

Recibido: $\quad 20.05 .10$

Aceptado: 28.06 .10

Sumario: 1. Introducción. 2. La crisis 2008-2009 en Uruguay. 3. La Ley General de Cooperativas. 4. La intercooperación durante la crisis: a) Nuevas cooperativas. b) Desarrollo de alianzas, mercado y fomento cooperativo. c) Participación en el sector cooperativo y de economía social, relacionamiento nacional e internacional. 5. La realidad de las cooperativas al 2009. 6. Conclusiones. Fuentes de información referencial.

Resumen: La presente colaboración, cuya temática central es exponer la forma, modo y oportunidad en que eventual o potencialmente operó la intercooperación como medio instrumental utilizado para respaldar -y superar en su caso- el impacto de la crisis económica mundial en el espacio geopolítico de la República Oriental del Uruguay, plantea los efectos y secuelas de dicha crisis en dicho país observándose, no obstante, la absoluta relatividad de aquel impacto en ese espacio y en la realidad cooperativa, la práctica inexistencia de casos específicos o constatables de intercooperación, en tanto no fueran estrictamente necesarios a aquella finalidad, las positivas previsiones legales contenidas en la nueva, reciente normativa cooperativa, en relación a la promoción de la intercooperación e integración cooperativas, algunas interesantes experiencias de desarrollo de cooperativas en el crítico período considerado (2008-2009), todo la cual se cierra con algunas consideraciones a modo de conclusión relacionadas con la aptitud de la intercooperación y de la integración como medios - y como fines- para el progreso del cooperativismo en lo económico y en lo social.

Palabras clave: Uruguay. Crisis y cooperativas. Intercooperación e integración cooperativa.

Abstract: The present contribution, which main thematic is to expose the way, form and opportunity in which intercooperation operated as an instrumental way, eventually or potentially, to back up -and overcome if it was needed - the impact of the worldwide economic crisis in the geopolitical space in Uruguay. This work considers effects and consequences of this crisis in the mentioned country, despite the relatively of that impact in the reality of 
cooperatives, the almost inexistence or verifiable cases of intercooperation, in order they were not necessary, the positive legal previsions that are part of the new cooperative normative, in relation with the intercooperative promotion and integration, some interesting experiences of cooperative development in the critical period between 2008 and 2009. This contribution closes with some conclusions that consider the intercooperative's aptitude and the integration's ways - and at the end - for the progress of cooperatives in economic and social issues.

Keywords: Uruguay. Crisis and cooperatives. Intercooperation and cooperative integration. 


\section{Introducción}

El objeto y enfoque de este trabajo es analizar cómo una situación crítica mundial incidió en las cooperativas uruguayas y el interrelacionamiento que hubo entre ellas como respuesta a la crisis.

Para formular tal análisis debemos pues, conocer cómo la crisis económica mundial incidió y afectó en su caso a Uruguay y frente a ella la eventual reacción del cooperativismo en general y de las cooperativas en particular.

En este trabajo primero haremos una breve reseña que pretende indicar la situación económica del Uruguay particularmente en los años 2008 y 2009, a través de la exposición de ciertos indicadores macroeconómicos, luego estudiaremos la nueva ley reguladora de las cooperativas, ya que la misma pudo incidir o incidió efectiva y directamente en las cooperativas nacionales y en su funcionamiento, atento a la incorporación de nuevas soluciones en la materia, y por último nos enfocaremos en las propias cooperativas y en las conductas y acciones de intercooperación que se llevaron en su caso adelante como reacción y respuesta a la situación planteada en los dos años objeto de estudio.

\section{La Crisis 2008-2009 en Uruguay}

Uruguay, a diferencia de lo que sucedió aparentemente en casi todos los países del mundo, prácticamente no sufrió, en términos generales, la crisis que comenzó en el año 2008. En efecto. A pesar de la crisis mundial y de los efectos cuasi ineludibles que deberían haber repercutido en el país, advertimos que durante el 2008 y el 2009 el PBI tuvo incluso un crecimiento moderado.

Si bien, al igual que los otros países de América Latina y el Caribe, Uruguay experimentó una relativa desaceleración de su crecimiento económico en 2009, logró mantener sin embargo un crecimiento positivo, de por lo menos $1.2 \%{ }^{1}$, con tendencia a un cierto incremento estimado del mismo.

En el área del mercado laboral observamos que también fue más positivo en Uruguay que en la mayoría de los países de la América Latina. En ese sentido, Uruguay y Perú fueron los países que tuvieron un decrecimiento de la tasa de desempleo, la cual bajó de $8.2 \%$ a $7.9 \%$ en los años 2008 y 2009. La fuerte creación de empleos en Uruguay

\footnotetext{
1 www.presidencia.gub.uy.
} 
fue el motivo principal que propulsó un mayor número de ocupados pasando de $57.1 \%$ a $58.3 \% .^{2}$

Frente a esta realidad, Uruguay tuvo, además, un aumento importante de los salarios medios reales (7.7\%). Es en apariencia el incremento más alto a nivel regional y es superior al incremento observado en 2008 (3.3\%).

Por otra parte, la inflación con significativa tendencia a la baja fue otro factor determinante para el aumento real de los salarios ya que la inflación acumulada en 2009 fue de $5.3 \%$ en octubre, en comparación con un $8 \%$ en similar periodo de $2008 .^{3}$

El Ministro de Economía y Finanzas del país, en declaraciones públicas $^{4}$, expresó que la cifra actual de deuda pública nominal neta a 2008, se fijaba en US\$ 8.255 millones, apenas $26 \%$ del Producto.

En lo que se refiere a la demanda interna, Uruguay creció a lo largo de este período tanto en inversiones como en el consumo privado.

Ante esta realidad analizaremos el crecimiento de las cooperativas y la interrelación entre las mismas durante el período 2008 y 2009.

\section{La Ley General de Cooperativas}

Antes de abordar el tema central de esta presentación, es importante hacer referencia a otro factor que incidió directamente en las cooperativas, su funcionamiento y su rol tanto en la sociedad como en la economía uruguaya: La nueva Ley General de Cooperativas.

En octubre de 2008 se sancionó una nueva ley en la materia que cambia el panorama y las perspectivas para las cooperativas. Se podría compartir la idea de que la ley procura mantener y hasta garantizar el conveniente equilibrio entre la autonomía de los cooperativistas y especialmente de las cooperativas mismas, por un lado, y el alcance del control estatal, normativo y operativo sobre aquellas por parte del Estado, por el otro. Como se podría compartir que los aspectos más significativos que se podrían destacar o podrían resultar de esta nueva ley lucirían, de acuerdo a sus antecedentes, los principios que la informan y las soluciones nucleares que ella consagra, entre otros, y con matices, los siguientes:

- Evitar la fragmentación del sector cooperativo e impulsar el reconocimiento de su unidad básica.

2 www.mef.gub.uy.

3 Id. cit., 2.

4 Id. cit., 2. 
- Generar certezas y seguridad jurídica para quienes contratan con las cooperativas.

- Alcanzar la congruencia entre las metas de las cooperativas orientadas hacia el desarrollo y de los asociados, así como la autosuficiencia.

- Facilitar la cooperación e integración cooperativas.

- Facilitar procesos de integración regional.

- Modernizar los aspectos empresariales de las cooperativas.

- Incrementar y mejorar los instrumentos de capitalización.

Consideramos que facilitar la intercooperación cooperativa y los procesos de integración regional son, en el contexto conceptual de este artículo de doctrina e investigación, dos puntos fundamentales de la ley, en tanto que tienden a promover justamente la intercooperación a desarrollar.

Ya desde las primeras disposiciones de la ley cooperativa se enuncia la «Cooperación entre cooperativas» como uno de los principios de observaniza obligatoria para todas la cooperativas, enunciado que luego se complementa, básicamente, de la manera siguiente:

- Por una parte, las normas que instituyen y regulan esquemas asociativos de carácter prevalente aunque no necesariamente sólo gremial, con acento en actividades de tipo representativo, defensa de intereses comunes y apoyos diversos a las cooperativas base (federaciones y confederaciones).

- Por otra parte, con disposiciones que contemplan fenómenos asociativos con objetivos generalmente económicos pero también con otros fines como la fusión en sus dos modalidades (por creación o por incorporación) o la constitución de cooperativas de segundo o ulterior grado, así como otras modalidades de cooperación económica como son, a título de sólo ejemplo (1) las «cooporaciones cooperativas», las que son definidas como "aquellas asociaciones empresariales que (...) tengan por objeto la definición de políticas empresariales, su control y, en su caso, la planificación estratégica de la actividad de sus socios, así como la gestión de los recursos y actividades comunes»; (2) las «cooperativas mixtas», considerándose tales «aquellas en que existen socios minoritarios cuyo derecho de voto (...) se podrá determinar (...) en función del capital aportado; y (3) las «secciones», que tienen por objeto desarrollar «actividades económico-sociales específicas, complementarias del objeto social principal» de una cooperativa dada.

- Por último, con soluciones orientadas a regular una persona pública no estatal, el Instituto Nacional del Cooperativismo 
(INACOOP), con fines esencialmente de promoción, y administrada por un directorio integrado por cinco personas designadas por el Poder Ejecutivo (3) y personas igualmente designadas por dicho poder del Estado pero a propuesta del movimiento cooperativo organizado en la Confederación Uruguaya de Entidades Cooperativas (ver infra) (2) y que cuenta, por un lado, con un director ejecutivo y por el otro, con un Consejo Consultivo del Cooperativismo integrado por representantes designados por cada una de las clases de cooperativas tipificadas en la ley (que son nueve: de trabajo, de consumo, agrarias, de vivienda, de ahorro y crédito, de seguros, de garantía recíproca, sociales, y de artistas y oficios conexos) y por dos representantes de la Universidad de la República (UDELAR) y dos de la Administración Nacional de Educación Pública (ANEP).

No es una solución menor en cuanto a novedad legislativa ciertas disposiciones de dicha ley que conceden un tratamiento preferencial a las cooperativas de trabajo en cuanto a resultar adjudicatarias de la explotación o propiedad de empresas o en crisis que se encuentran en situaciones concursales previstas en una ley de fecha contemporánea aunque unuméricamente anterior a la ley cooperativa, como es una ley que regula el concurso y la reorganización empresarial de aquéllas, de manera orgánica e integral y que incluye igualmente aquel trato preferencial: ley concursal, n. ${ }^{\circ} 18.387$, ley cooperativa, ley n. ${ }^{\circ} 18.411$.

\section{La intercooperación durante la crisis}

Para estar en mejores condiciones de analizar el desarrollo de la intercooperación o, si se prefiere, del intercooperativismo durante estos dos años de objeto y alcance del presente estudio, haremos una subdivisión tridimensional mere metodológica por áreas temáticas:

\section{a) Nuevas cooperativas}

El último relevamiento de las cooperativas uruguayas fue realizado por la Confederación Uruguaya de Entidades Cooperativas (CUDECOOP) —entidad asociativa de derecho privado que nuclea en su seno a las prácticas totalidad de las cooperativas del país- en 1999. En ese entonces, la suma de los padrones por entidades sociales era de más de 1.200 cooperativas que significaban una cifra superior a los 
850.000 afiliados, aunque es importante tener en cuenta que muchos uruguayos pertenecen a más de una organización cooperativa a la vez.

Estos números nos sirven de base para comprender si hubo o no un crecimiento considerable de cooperativas durante el período 20082009.

Nos consta que se han desarrollado nuevas cooperativas en estos últimos años, fomentadas en su mayoría por el Estado a través de los Ministerios de Vivienda, Ordenamiento Territorial y Medio Ambiente, (cooperativas de vivienda) y de Desarrollo Social (cooperativas sociales).

El gobierno nacional, a través de una alianza con la Federación de Cooperativas de Producción del Uruguay, afiliada a la CUDECOOP, buscó, a través de visitas a cooperativas de la capital y del interior del país sensibilizar a sus integrantes en la posibilidad de llevar adelante nuevos y más emprendimientos para el desarrollo nacional.

No debería extrañar tal alianza en la medida que la normativa aplicable a las cooperativas sociales se remite, tanto en forma principal como subsidiaria, a la dispuesta para las cooperativas de trabajo, aunque con las especificidades de la normativa propia de las primeras

Las llamadas cooperativas sociales en particular — que ya estaban contempladas en una legislación anterior a la Ley General y que proporcionan un puesto de trabajo a sus socios para el desarrollo de actividades económicas y tienen la finalidad de beneficiar a «jefes y jefas de hogares pertenecientes a sectores con necesidades básicas insatisfechas, jóvenes, discapacitados, minorías étnicas y todo grupo en situación de extrema vulnerabilidad social»— son, tal vez las que más se han desarrollado durante este período. El MIDES (Ministerio de Desarrollo Social), a través de su Unidad de Cooperativas Sociales procuró asesorar a las cooperativas en las áreas de negocios e inserción, así como fomentó el desarrollo de nuevas cooperativas de la siguiente manera: estudio de la maduración del grupo y de la idea empresarial con información, estudios de viabilidad social y económica, aprobación del MIDES, capacitación de los cooperativistas y, la firma de los estatutos. Tales cooperativas suman una cantidad estimada de 109 cooperativas $^{5}$ que nuclean un total de 1090 cooperativistas. A través de CUDECOOP y dicho Ministerio se procuró justamente la intercooperación con y entre las cooperativas sociales y un logro importante fue que las mismas pudieron prestar sus servicios al Estado mediante la celebración de por lo menos 84 contratos con el MIDES6 6 .

5 www.mides.gub.uy.

6 Id. cit., 5. 


\section{b) Desarrollo de alianzas, mercado y fomento cooperativo}

Durante el período considerado se enfatizó tanto la generalización de alianzas para recibir recursos como para resolver problemas en conjunto, ámbito de actuación en que se pretendió mejorar la relación entre ciertos actores sociales: entre el Estado, los técnicos, los productores y los consumidores.

Por intermedio o mediante la realización de varios y diferentes encuentros entre cooperativistas se persiguió y se produjo el intercambio de ideas, la definición de políticas cooperativas y el diseño y ejecución de planes para seguir desarrollándose; sin embargo no hubieron, salvo en el Día Internacional de las Cooperativas del año 2009, eventos especiales referidos a la crisis en sí misma, así como tampoco ha habido eventos que signifiquen un interrelacionamiento proactivo entre cooperativas de diferentes sectores y áreas de desempeño.

El Ministerio de Vivienda, Ordenamiento territorial y Medio Ambiente (MVOTMA), por su parte, concedió créditos para la construcción de viviendas a 322 familias cooperativistas de ayuda mutua. Éstas fueron las primeras adjudicaciones que se realizaron con la nueva reglamentación de las cooperativas. Este proceso establece etapas, las cuales comienzan con la inscripción, luego tres etapas ligadas a la viabilidad del terreno y a la aprobación del anteproyecto. Cumplidos estos pasos, se realiza un sorteo, siempre y cuando quienes culminen todas las etapas sean más que los créditos que se otorgan. El MVOTMA aclaró en su momento que se apuesta a invertir en el sector cooperativo para que él mismo empiece a invertir y darle forma a Uruguay. $^{7}$

c) Participación en el sector cooperativo y de Economía Social, relacionamiento nacional e internacional

A través de la Confederación Uruguaya de Entidades Cooperativas (CUDECOOP), del Centro de Desarrollo Cooperativo, del Centro Cooperativista Uruguayo (CCU), de la Unidad de Estudios Cooperativos (UEC) de la Universidad de la República y de las diferentes federaciones de cooperativas se ha mantenido el concepto de fomentar la intercooperación, el intercooperativismo a través de planes, cursos, seminarios

\footnotetext{
7 www.larepublica.com.uy/economia/364223-impulso-al-desarrollo-cooperativo.
} 
y actividades que impliquen el intercambio de experiencias de las cooperativas, como mencionamos anteriormente.

Uno de los objetivos de la nombrada UEC es precisamente fortalecer el relacionamiento con el sector cooperativo a través del desarrollo de iniciativas de trabajo en conjunto con las entidades. Otra de las metas de dicha Unidad es «desarrollar una estrategia de relacionamiento con el exterior, que permita fortalecer las relaciones sur-sur y generar condiciones para la cooperación norte-sur, en la concreción de proyectos conjuntos que al tiempo que consoliden la unidad con recursos extra presupuestales permitan su proyección internacional» 8 .

Durante el 2008 y el 2009 ha habido numerosos encuentros y seminarios organizado por cooperativas, organizaciones, el Estado y otras instituciones, con el fin de crear centros de encuentro entre cooperativistas. No obstante, no hubieron o no son notorios ni conocidos eventos especiales que fomenten el intercooperativismo para salir de la crisis que afectó al mundo y en su caso al Uruguay.

Ello, a pesar de que, en el resto del mundo, en el Día Internacional del Cooperativismo del año 2009, el tema que se trató, y que propuso la Alianza Cooperativa Internacional (ACI) apuntó justamente a la contribución del cooperativismo a la salida de la crisis internacional.

Ahora bien. Visto los sucesos económico-sociales a nivel mundial y comparando la situación internacional con la local, corresponde que reconozcamos y señalemos que el cooperativismo uruguayo contribuyó a conservar una economía que no resintiera ni sufriera tanto el impacto de la crisis global. Es un hecho constatable que las cooperativas de producción o de trabajo, a modo de ejemplo, conservaron proporcionalmente más puestos de trabajo que otros tipos empresariales, como lo es que desde las cooperativas de vivienda y otras ramas del quehacer cooperativo ensayaron estrategias para la producción de alimentos y el abastecimiento a los sectores carentes ${ }^{9}$.

Adicionalmente. Las cooperativas de ahorro y crédito, básicamente las Ilamadas «de capitalización» mantuvieron la cadena de pagos en zonas del interior del país, incluso en aquellas en las que habían desaparecido todos los grandes bancos de red, y refinanciaron a los productores rurales y a las pequeñas y medianas empresas ${ }^{10}$.

8 www.extension.edu.uy.

9 www.cofac.com.uy.

10 Id. cit., 9. 


\section{La realidad de las cooperativas al 2009}

La Comisión Honoraria del Cooperativismo (CHC) —antecedente institucional de la actual DINACOOP_ funcionaba en la órbita de la Oficina de Planeamiento y Presupuesto de la Presidencia de la República y era un órgano asesor del Poder Ejecutivo en materia cooperativa y un espacio de relacionamiento del Sector Cooperativo con el Estado.

La CHC tenía entre sus funciones «proyectar el Plan Nacional de Desarrollo Cooperativo en coordinación con los representantes de los ministerios competentes para cada rama, dando seguimiento a su ejecución».

La CHC había priorizado esta tarea y había ampliado la convocatoria a los gobiernos locales, actores cooperativos y a la sociedad civil interesada en el desarrollo de propuestas cooperativas y asociativas.

Ahora bien. Es un hecho que la cooperativa afecta e incide en el entorno. Es que es un fenómeno importante en lo social y en lo solidario. Se ha entendido que para crear un impacto positivo de las cooperativas es necesario hacer economía y ser una escuela de participación. Se considera, sin embargo, que hay ausencia de preparación porque no hay suficiente ni adecuada cultura cooperativa, ni en primaria ni en secundaria. Frente a la realidad de las cooperativas se plantea:

- La profundización de la formación y la dimensión social en cooperativismo.

- La incorporación del cooperativismo al sistema educativo y la formación de técnicos especializados.

- La mejora en la exigencia, por parte de las autoridades públicas, de cumplimiento de ciertos criterios para denominarse cooperativa.

- El involucramiento de la CHC en el control al sector cooperativo.

- En el corto plazo: la administración de tiempos y recursos para el mediano y largo plazo ${ }^{11}$.

Durante el evento denominado "Seminario: II Reunión Regional "Hacia el Plan Nacional de Desarrollo Cooperativo"» y según el informe expuesto en la oportunidad ${ }^{12}$ durante el evento se enfatizó en los siguientes puntos:

- La importancia de articularse con el fin de conocerse, comercializar, capacitarse y obtener financiamientos.

- Para lograr una articulación eficiente es importante que la capacitación sea de excelente calidad y que tanto sus expositores

11 www.chc.gub.uy.

12 Id. cit., 11. 
como los participantes acudan a ella con el fin real de aprender y no para tan sólo escuchar. Además se concluyó que falta capacidad de gestión para una capacitación óptima. Consideraron que en la actualidad existen ineficiencias del sistema cooperativo y por ende, se debe profundizar el desarrollo en calidad y en eficiencia, siendo el desarrollo distinto al crecimiento.

- Entre los objetivos que se propusieron está el conocerse más entre las cooperativas, interrelacionarse con los diferentes organismos del Estado, elaborar un banco de datos que incluya información sobre los grupos, con los antecedentes, los apoyos recibidos y la ubicación.

- Además resaltaron el hecho de mejorar el sistema productivo para facilitar las articulaciones.

- Estimular nuevos emprendimientos (viables tanto social como económicamente). Crear mayor cantidad de acuerdos de empresas cooperativas del mismo sector. Integrar cooperativas de distintas modalidades para explotar un sector de negocios determinado.

- Generar integración a nivel local y regional entre cooperativas consiguiendo financiación para la evaluación y desarrollo de esas experiencias.

- Consideraron importante obtener elementos que posibiliten hacer un Balance social de impacto de las cooperativas, a través de estudios que permitan evaluar los aportes del movimiento a la comunidad.

- Incentivar la formación especializada de dirigentes y funcionarios para llegar a una profesionalización de los mismos.

- Construir un modelo de gestión económica y social propia de las cooperativas.

\section{Conclusiones}

La crisis económica mundial que conllevó otros problemas, incluso sociales, como desempleo, de acuerdo a la limitada información disponible, no fue ni resultó de alto impacto en Uruguay, que, como ya señalamos, tuvo durante los dos años 2008 y 2009, un PBI hasta con crecimiento, crecimiento moderado, pero igualmente destacable.

Las cooperativas siguieron desarrollándose, acentuándose una mayor y hasta mejor relación con el Gobierno Nacional. Además, siguieron organizándose eventos que significaran una unión de las cooperativas para, aunque fuera, intercambiar experiencias. 
La nueva Ley General de Cooperativas favorece a las mismas ya que impulsa el desarrollo y la integración de las cooperativas a nivel nacional y regional, entre otros objetivos y resultados.

Frente a esta realidad las cooperativas en Uruguay son actualmente más en número y diversidad, y están adquiriendo significativamente mayor protagonismo en la sociedad.

La intercooperación, por ende, no fue en esas circunstancias el eje protagónico alrededor del cual giró el cooperativismo como forma instrumental indispensable para salir adelante y superar la crisis que afectó aunque sólo relativa y limitadamente en ciertos, puntuales sectores de la economía, al Uruguay, a la vez que, hasta donde consta, no se fomentaron encuentros tácticos o estratégicos entre cooperativas de diferentes sectores, seguramente por no ser aquellos estrictamente necesarios, ni hubieron, hasta donde también consta, acuerdos por lo pronto conocidos o significativos entre cooperativas y federaciones para unir fuerzas con el fin de mejorar sus respectivas perspectivas, ello sin perjuicio de eventuales encuentros o acuerdos en el ámbito regional del MERCOSUR.

Las diferentes instituciones vinculadas con el cooperativismo como las arriba mencionadas - CUDECOOP, UEC, CHC, Centro de Desarrollo Cooperativo, CCU— mantuvieron sus líneas propias de acción sin poner un énfasis particular en el intercooperativismo como medio indispensable o meramente conveniente para salir de la crisis económica.

Cabe resaltar que según la OIT13, a nivel mundial, el impacto que tuvo la crisis sobre las cooperativas fue menos fuerte en comparación con el soportado por otros tipos de empresas privadas. Un ejemplo, fue, cómo los bancos cooperativos enfrentaron un aumento de solicitudes de afiliación y de depósitos de ahorros. Este repentino crecimiento seguramente dificultó su operativa pero seguramente también redujo su vulnerabilidad y aumentó la transparencia, aunque corresponde señalar que en Uruguay no existen tales bancos, sólo algunas cooperativas de ahorro y crédito de intermediación financiera que desarrollan similares actividades que los llamados «bancos» y un número significativo de cooperativas de ahorro y crédito de capitalización.

Ante esta realidad las cooperativas pasan a ser un modelo alternativo en lo asociativo, desde el punto de vista económico y social, pese a que la sustentabilidad y rentabilidad de las mismas pasan a tener plazos más largos de maduración.

Podemos concluir, entonces, que las cooperativas más allá de la crisis económica o de la estabilidad de un país, son una opción formal y

13 www.ilo.org. 
sustancial de emprendimientos o negocios, cada vez más usual y fiable, especialmente se acentúan los procesos de intercooperación entre las mismas mediante su interrelación, interacción e interdependencia, tanto a nivel nacional como regional e internacional, para lo cual, en el caso particular de Uruguay, la instrumentación, uso y aplicación oportunas y adecuadas de las herramientas de cooperación e integración previstas en su reciente Ley General de Cooperativas, tienen la aptitud de contribuir al logro de aquella intercooperación, tanto como medio o como fin.

\section{Fuentes de información referencial}

www.ccea.org.uy/ccea_nws04/docs/decreto558.pdf www.rau.edu.uy/sui/publicaciones/algunosTopicos/doc_tr22.pdf www.extension.edu.uy/programas/uec www.larepublica.com.uy/economia http://www.fcpu.coop/ www.mides.gub.uy www.chc.gub.uy www.presidencia.gub.uy www.mvotma.gub.uy http://listas.chasque.net/pipermail/vecinet/2004-July/date.html\#119 www.cofac.com.uy www.ilo.org www.fucac.com.uy www.neticoop.org.uy 